\title{
Domestication du gros baume (Hyptis suaveolens (L.) Poit.) : techniques de production et potentiels insectes ravageurs au sud du Bénin
}

\author{
L. E. AHOTON ${ }^{1}$, T.B.C. ALAVO ${ }^{2 *}$, M. A. AHOMADEGBE ${ }^{2}$, C. AHANHANZO ${ }^{2}$ et \\ C. AGBANGLA ${ }^{2}$
}

\author{
${ }^{1}$ Faculté des Sciences Agronomiques, Université d'Abomey-Calavi, Benin. \\ ${ }^{2}$ Faculté des Sciences et Techniques, Université d'Abomey-Calavi, Benin. \\ "Corresponding author, E-mail: thieryalavo@hotmail.com, BP: 215 Goomey, Benin.
}

\section{RESUME}

Hyptis suaveolens Poit. est une herbe vivace de la famille des Lamiaceae qui pousse en colonies denses en région tropicale. Elle est originaire de l'Amérique tropicale mais se retrouve aujourd'hui sous forme de mauvaise herbe un peu partout dans le monde. C'est une plante qui dégage une odeur caractéristique similaire à la menthe lorsqu'elle est broyée. Diverses études ont montré que Hyptis suaveolens a un effet répulsif contre les moustiques, un effet antifongique, antibactérien, anti-plasmodium et anticonvulsivant. L'importance de cette plante pourrait conduire à son exploitation industrielle. Il est donc nécessaire de rechercher les meilleures techniques culturales pour une production massive de cette plante. Le présent travail a évalué l'effet de différentes techniques culturales, à savoir : labour, non-labour, semis direct et repiquage sur la croissance et le rendement de cette plante. Les paramètres comme la hauteur des plantes, le nombre de branches végétatives, le nombre d'entrenoeuds sur la branche principale, la longueur de la première branche végétative et la biomasse fraîche des feuilles ont été analysés 1 et 2 mois après semis. Par ailleurs, les différentes espèces d'insectes se nourrissant sur la plante au cours des essais ont été déterminées. Les techniques comme le labour avec semis direct et le non-labour avec semis direct ont donné les meilleurs résultats. Il est recommandé d'utiliser la technique de non-labour avec semis direct pour la production de Hyptis suaveolens, étant donné que cette technique nécessite moins d'effort et donne un rendement équivalent à celui du labour avec semis-direct. Zonocerus variegatus, Cosmophila flava, Acreaa zetes, Bemisia tabaci sont les espèces d'insectes ravageurs identifiées sur cette plante au cours des travaux.

(C) 2010 International Formulae Group. All rights reserved.

Mots clés : Hyptis suaveolens, domestication, techniques culturales, insectes ravageurs.

\section{INTRODUCTION}

Le gros baume (Hyptis suaveolens Poit.) est une herbe vivace de la famille des Lamiaceae qui pousse sauvagement en colonies denses le long des rues, dans les jardins et les brousses en région tropicale. C'est une plante d'environ $2 \mathrm{~m}$ de haut qui dégage une odeur caractéristique similaire à la menthe lorsqu'elle est broyée. H. suaveolens est originaire de l'Amérique tropicale mais se retrouve aujourd'hui sous forme de mauvaise herbe un peu partout dans le monde (Chukwujekwu et al., 2005). C'est une plante qui possède des feuilles simples, entières, opposées, pétiolées, ovales, pubescentes sur les deux faces (Coates et Ayerza, 1995).

Des travaux réalisés sur l'huile essentielle de $H$. suaveolens, ont révélé que 
l'huile essentielle de cette plante chasse effectivement les moustiques et peut-être utilisée comme moyen de lutte anti-vectorielle contre le paludisme (Abagli et al., 2010; Jaenson et al., 2006).

De plus, un certain nombre de travaux ont révélé l'effet antifongique, antibactérien, anti-plasmodium et anticonvulsivant des huiles essentielles de cette herbe (Krishnamurthy et Shashikala, 2006; Chukwujekwu et al., 2005; Zigler et al., 2002; Azevedo et al., 2001). Compte tenu de l'importance médicinale de cette plante, on pourrait penser à son exploitation industrielle, les années à venir. Il est donc nécessaire de rechercher les meilleures techniques culturales pour une production massive de $H$. suaveolens.

Par conséquent, l'objectif du présent travail est d'identifier les meilleures techniques culturales permettant d'obtenir un meilleur rendement de $H$. suaveolens. Dans cet ordre d'idée, il a été aussi recensé et identifié les différents insectes ravageurs présents sur cette plante au cours de cette étude.

\section{MATERIEL ET METHODES}

\section{Site expérimental}

Le travail a été conduit à la Ferme d'Application et de Production (FAP) de la Faculté des Sciences Agronomiques (FSA, Altitude $\left.17,4 \mathrm{~m}, 06^{\circ} 24^{\prime} \mathrm{N}, 02^{\circ} 20^{\prime} \mathrm{E}\right) \mathrm{de}$ l'Université d'Abomey-Calavi (AbomeyCalavi, Bénin). Le climat dans la zone d'étude est de type subéquatorial et caractérisé par deux saisons de pluies et deux saisons sèches.

\section{Matériel végétal}

Les semences de $H$. suaveolens utilisées dans le cadre de cette étude ont été récoltées à Ayédèro (Arrondissement de Dassa-Zoumé) situé à environ $205 \mathrm{~km}$ au nord de Cotonou. Elles ont été récoltées en mars 2008. Le poids de 100 graines de cette espèce est en moyenne de 0,4 gramme.
Collectes des données relatives à la production végétale

Deux formes du travail du sol ont été testées, à savoir: le labour (sous forme de billons) et le non labour (poquets). Les parcelles sont d'abord sarclées à la houe (comme généralement exécutées par les paysans au sud Bénin). En ce qui concerne les modalités d'installation des plantes sur les parcelles, elles sont au nombre de deux : le semis direct et le repiquage.

Le semis a été réalisé le 05 mai 2008 sur les parcelles labourées et non labourées (poquets) à raison de huit à dix graines par poquet. Quinze jours après semis, les plantules en excès provenant du démariage des plantes des parcelles labourées et non labourées ont été repiquées sur les mêmes types de parcelles (parcelles labourées et parcelles non labourées). Chaque poquet contient après démariage et repiquage deux plantes. Le taux de germination calculé à partir des semis réalisés sur les parcelles est de $97,8 \%$. L'essai a été conduit sur un sol ferralitique sur lequel des bœufs ont été précédemment élevés pendant 3ans. Le précédent cultural est constitué de chaumes issus de la culture de maïs installée au cours de la grande saison pluvieuse. Les parcelles sont régulièrement sarclées à la houe tout au long de l'expérimentation. Le dispositif expérimental utilisé est le bloc aléatoire complet, avec quatre blocs et quatre répétitions. Chaque bloc comprend quatre unités expérimentales ayant chacune une surface de $18 \mathrm{~m}^{2}$, soit quatre lignes de $10 \mathrm{~m}$ x 0,6 m. La distance entre lignes est de 0,6 $\mathrm{m}$ et entre plantes de $0,4 \mathrm{~m}$. Chaque ligne comporte 52 plantes à raison de deux plantes par poquet soit 83350 plantes par hectare. L'essai s'est déroulé du 05 mai au 07 août 2008.

Les paramètres suivants ont été déterminés sur dix (10) plantes au niveau des deux lignes centrales de chaque répétition:

- Hauteur des plantes un et deux mois après semis; 
- Nombre moyen de branches végétatives un mois après le semis;

- Nombre d'entrenoeuds sur la branche principale un mois après semis;

- Nombre de feuilles après un mois;

- Production de biomasse fraîche après deux mois.

Pour déterminer la biomasse fraîche, les feuilles ont été récoltées deux mois après semis. Celles-ci ont été pesées immédiatement après récolte à l'aide de la balance électronique Denver Maxx (Société Denver, Allemagne). La biomasse fraîche par hectare a donc été calculée en considérant le nombre total de plantes semées à l'hectare.

\section{Inventaire des insectes ravageurs}

Le champ de $H$. suaveolens est inspecté à intervalle d'une semaine pendant 8 semaines consécutives. Les plantes situées dans les deux diagonales du champ sont observées minutieusement. Les ravageurs rencontrés sont identifiés sur place s'ils sont connus et les dégâts causés aux plants sont reportés. Les chenilles difficiles à identifier sur place sont élevées dans une cage sur la plante hôte jusqu'au stade adulte. Les imagos issus de ces chenilles sont identifiés à l'aide d'une clé d'identification.

\section{Analyse statistique}

Les valeurs moyennes des différentes variables collectées ont été calculées. Le caractère significatif ou non des différences notées est évalué par une analyse de variance (ANOVA). Le classement des moyennes est réalisé par le test de Newman et Keuls. Le modèle d'analyse de la variance utilisé est le modèle mixte croisé à trois facteurs : un facteur aléatoire (bloc) et deux facteurs fixes, à savoir : labour et semis.

\section{RESULTATS}

Taille des plantes et nombre de feuilles après un mois de végétation

Le Tableau 1 indique les valeurs moyennes enregistrées en ce qui concerne la taille des plantes et le nombre de feuilles après un mois de culture.
La hauteur des plantes de $H$. suaveolens après un mois de culture varie de $14,10 \mathrm{~cm}$ à $21,09 \mathrm{~cm}$. L'analyse statistique montre qu'il existe une différence significative au seuil de $5 \%$ entre les traitements. Le traitement non labour (poquet) avec semis direct (T3) a donné la meilleure croissance en hauteur. Ceci peut être dû au fait qu'au cours du semis, les trous réalisés sous forme de poquet en accumulant plus d'eau d'arrosage et de pluies maintiennent une certaine humidité autour des plantes. Cette humidité favoriserait la croissance et le développement des plantes. Il est remarqué que les plantules se trouvant sur les billons provenant des semis n'ayant pas ces trous se fanent plus rapidement que les autres surtout les après midi de fort ensoleillement.

En ce qui concerne le nombre de feuilles émises par les plantes, l'analyse statistique a montré qu'il existe une différence significative au seuil de $5 \%$ entre les traitements. De l'analyse du Tableau 1, il ressort que le labour avec repiquage de plantules, le labour et le non labour (poquet) avec semis direct ont donné des valeurs plus élevées en nombre de feuilles que le non labour avec repiquage. Le semis direct a donné les valeurs les plus élevées en matière du nombre de feuilles émises par la plante. Le poquet avec semis direct et le labour avec semis direct, ont induit un nombre similaire de feuilles émises par les plantes, soit respectivement 54,085 et 59,88 .

\section{Nombre d'entrenoeuds sur la tige principale et le nombre de branches végétatives}

Le Tableau 2 présente les résultats obtenus pour ces deux paramètres.

Le nombre moyen d'entrenoeuds après un mois de croissance varie de 4,82 à 5,95 (Tableau 2). L'analyse statistique montre qu'il existe une différence significative au seuil de 5\% entre les traitements réalisés. Le non labour (poquet) suivi d'un repiquage des plantules est le traitement qui a donné le nombre le plus 
faible d'entrenoeuds $(4,82)$. Pour les autres traitements ces valeurs sont similaires.

En ce qui concerne le nombre moyen de branches végétatives, l'analyse statistique montre qu'il n'existe pas une différence significative entre les traitements.

Taille des plantes et biomasse fraîche des feuilles après deux mois de végétation

Après deux mois de culture, la taille moyenne des plantes varie de 79,61 à 95,73 $\mathrm{cm}$. L'analyse statistique montre une différence significative entre les traitements. Le traitement non labour (poquet) avec semis direct (T3) a maintenu son avance de croissance sur les autres après deux mois de culture. Il a la valeur la plus élevée, soit $95,73 \mathrm{~cm}$ (Tableau 3).

En ce qui concerne la biomasse fraîche, l'analyse statistique montre qu'il n'existe pas une différence significative entre les traitements. Néanmoins, les traitements comme non labour avec semis direct (T3) et labour avec semis direct (T4) ont les valeurs les plus élevées par rapport aux deux autres traitements. $\mathrm{Si}$ nous comparons les quatre valeurs obtenues à la première récolte en ce qui concerne la biomasse fraîche au niveau de tous les traitements, le traitement labour avec semis direct a la plus grande valeur. Ceci s'expliquerait par le fait que le labour a ameubli le sol et a permis aux racines, en s'enfonçant plus profondément, de mettre à la disposition des plantes de ce traitement plus d'éléments nutritifs.

Insectes ravageurs rencontrés sur les plants de Hyptis suaveolens

Zonocerus variegatus (Orthoptera:

Pyrgomorphidae)

Ce criquet était abondant dans le champ et se nourrit des feuilles de $H$. suaveolens. Les jeunes plants étaient complètement détruits par ce ravageur qui consomme entièrement toutes les premières feuilles et le bourgeon terminal.

Anomis (Cosmophila) flava (Lepidoptera: Noctuidae)

Les chenilles de cette espèce se nourrissent des feuilles de $H$. suaveolens et provoquent des perforations du limbe.

Acraea zetes (Lepidoptera: Acraeidae)

Les chenilles de cette espèce rongent les parties supérieures des feuilles de $H$. suaveolens.

Bemissia tabaci (Homoptera: Aleurodidae)

Des colonies plus ou moins denses de ces mouches blanches étaient présentes sur les feuilles de $H$. suaveolens. Les mouches blanches font d'innombrables piqûres sur les feuilles et sont capables de transmettre des virus à la plante.

Tableau 1 : Taille moyenne des plantes et le nombre moyen de feuilles émises par les plantes de chaque traitement après un mois de végétation.

\begin{tabular}{lcc}
\hline Traitements & $\begin{array}{c}\text { Taille moyenne de } \\
\text { la plante après un } \\
\text { mois de culture }(\mathbf{c m})\end{array}$ & $\begin{array}{c}\text { Nombre moyen de } \\
\text { feuilles après un } \\
\text { mois de culture }\end{array}$ \\
\hline Labour + repiquage $(\mathrm{T} 1)$ & $14,63 \mathrm{~b}$ & $41,97 \mathrm{ab}$ \\
Non labour+repiquage $(\mathrm{T} 2)$ & $14,10 \mathrm{~b}$ & $34,53 \mathrm{~b}$ \\
Non labour+semis direct & $21,09 \mathrm{a}$ & $54,05 \mathrm{ab}$ \\
$(\mathrm{T} 3)$ & $19,83 \mathrm{a}$ & $59,88 \mathrm{a}$ \\
Labour + semis direct (T4) & $7,37 *$ & $4,49 *$ \\
\hline Probabilité & 15,07 & 22,74 \\
\hline Cv\% & & \\
\hline$*:$ Différence significative au seuil de $5 \% ;$ les moyennes suivies de la même lettre ne sont pas statistiquement \\
différentes. Cv\% = Coefficient de variation
\end{tabular}


Tableau 2 : Nombre moyen d'entrenoeuds et de branches végétatives par traitement après un mois de culture.

\begin{tabular}{lcc}
\hline Traitements & $\begin{array}{c}\text { Nombre moyen } \\
\text { d'entrenoeuds après } \\
\text { un mois de culture }\end{array}$ & $\begin{array}{c}\text { Nombre moyen de branches } \\
\text { végétatives après un mois de } \\
\text { culture }\end{array}$ \\
\hline T1 & $5,10 \mathrm{ab}$ & $10,50 \mathrm{a}$ \\
$\mathrm{T} 2$ & $4,82 \mathrm{~b}$ & $9,62 \mathrm{a}$ \\
$\mathrm{T} 3$ & $5,90 \mathrm{a}$ & $11,80 \mathrm{a}$ \\
$\mathrm{T} 4$ & $5,95 \mathrm{a}$ & $11,67 \mathrm{a}$ \\
\hline Probabilité & $4,57 *$ & $3,83 \mathrm{~ns}$ \\
\hline Cv\% & 9,75 & 9,68 \\
\hline T1 = labour + repiquage ; T2 = non labour (poquet) + repiquage ; T3 = non labour (poquet) + semis direct ; T4 $=$ \\
labour + semis direct. Cv\% = Coefficient de variation \\
ns : différence non significative au seuil de 5\% ; : différence significative au seuil de 5\% ; les moyennes suivies de la \\
même lettre ne sont pas statistiquement différentes.
\end{tabular}

Tableau 3 : Taille moyenne des plantes et biomasse moyenne des feuilles fraîches après deux mois de culture.

\begin{tabular}{lccc}
\hline Traitements & $\begin{array}{c}\text { Taille moyenne des } \\
\text { plantes après deux } \\
\text { mois de culture }(\mathbf{c m})\end{array}$ & $\begin{array}{c}\text { Biomasse } \\
\text { moyenne des } \\
\text { feuilles fraîches } \\
\text { après deux mois } \\
\text { de culture }(\mathbf{g})\end{array}$ & $\begin{array}{c}\text { Production de } \\
\text { feuilles en tonne } \\
\text { (t) par hectare } \\
\text { après la première } \\
\text { récolte }\end{array}$ \\
\hline T1 & $\begin{array}{c}117,58 \text { a } \\
\text { T2 }\end{array}$ & 111,04 a & 0,98 \\
T3 & $81,60 \mathrm{~b}$ & 144,15 a & 0,92 \\
T4 & 95,73 a & 150,58 a & 1,201 \\
\hline Probabilité & $91,33 \mathrm{a}$ & $2,95 \mathrm{~ns}$ & 1,255 \\
\hline Cv\% & $8,50 *$ & 17,31 & - \\
\hline ns : différence non significative au seuil de $5 \% ; *:$ différence significative au seuil de $5 \% ;$ les moyennes suivies \\
de la même lettre ne sont pas statistiquement différentes. \\
Cv\% = Coefficient de variation
\end{tabular}

\section{DISCUSSION}

La présente étude est le tout premier travail scientifique sur l'élaboration des itinéraires techniques de $H$. suaveolens. Elle a permis d'identifier les pratiques agricoles pouvant conduire à un bon rendement de cette plante. En effet, les traitements réalisés ont permis d'obtenir en deux mois de culture une première récolte en biomasse fraîche foliaire variant de $900 \mathrm{~kg}$ à $1255 \mathrm{~kg}$ par hectare. Le traitement non labour (poquet) avec semis direct (T3) et le labour avec semis direct (T4) ont donné les meilleurs rendements en matière de production de feuilles à l'hectare. Pour avoir une meilleure production de feuilles, la pratique de semis direct de cette plante est conseillée. En effet, cette pratique de non labour avec des semis réalisés sous forme de poquets permet dès la levée de la plante de mettre à la disposition de celle-ci plus d'eau. Les poquets de semis des parcelles non labourées réalisés sous forme de trous stockent plus d'eau que ceux des semis des parcelles labourées permettant ainsi un enracinement plus rapide et plus profond. 
Plus l'enracinement est dense et profond, plus faibles seront les pertes en eau par drainage dans la zone de sol exploitée par les racines et plus longues seront les périodes d'utilisation de cette eau par la plante (Scopel, 1999). Les résultats obtenus dans le cadre de ce travail sont en accord avec cette assertion. Au cours de notre étude nous avons remarqué que les jeunes plantes de $H$. suaveolens sont très sensibles au manque d'eau. En effet, les traitements (T1, T2 et T4) ne permettant pas un stockage minimum d'eau aux plantules entraînent une fanaison de ces dernières au cours des périodes de fortes chaleurs, ce qui limite la photosynthèse et de ce fait leur bonne croissance et développement.

En ce qui concerne le travail du sol (labour), celui-ci complète les actions du climat, des micro-organismes et des racines des plantes. En favorisant l'approvisionnement en eau, l'enfouissement des débris végétaux, l'ameublissement du sol et une meilleure circulation de l'air dans le sol, le labour contribue à améliorer l'état végétatif des plantes (Soltner, 1994). La production de biomasse foliaire un peu plus élevée $(1255 \mathrm{~kg})$ obtenue au niveau du traitement 4 (T4) s'explique par ces effets.

La biomasse de feuilles fraîches obtenue au niveau des traitements (T1 et T2) impliquant le repiquage des plantules est plus faible par rapport aux traitements T3 et $\mathrm{T} 4$ où la pratique de semis direct des graines est utilisée. Le temps de reprise des plantules et le choc de transplantation enregistré par ces dernières sont deux facteurs qui retardent la croissance et le développement de $H$. suaveolens, surtout si le repiquage est effectué en début de saison pluvieuse où le manque d'eau est le facteur le plus limitant. En matière de repiquage des plantules de $H$. suaveolens, le paramètre le plus important est l'âge de ces dernières. L'optimum est de 10 à 15 après semis. Au-delà, il existe une corrélation négative entre l'âge des plantules, le taux de reprise et le potentiel de production en feuille.
En matière de rentabilité, la comparaison des quatre traitements montre que le non labour avec semis direct est le système cultural le plus avantageux et le plus rentable économiquement car utilisant moins de main-d'œuvre et d'opérations culturales à réaliser.

Zonocerus variegatus, Anomis flava, Acraea zetes et Bemissia tabaci sont les ravageurs rencontrés et identifiés sur les plants de $H$. suaveolens au cours de la présente étude. Ces ravageurs ont causé des dégâts à divers degrés, mais les plus importants dégâts ont été causés par le criquet Zonocerus variegatus. Les dégâts causés par ces criquets sont très importants étant donné qu'ils détruisent entièrement la grande majorité des jeunes plants dès la levée. Ce qui nous obligeait à refaire les semis et les repiquages en vue d'assurer une densité normale des plants. Par conséquent, il va falloir penser à une stratégie de lutte contre le criquet Zonocerus variegatus sur H. suaveolens.

Les dégâts causés par les autres ravageurs ne paraissent pas trop importants au cours de la présente étude. Néanmoins, sachant que l'intensification de la production végétale conduit généralement à l'augmentation du nombre des ravageurs, il est à recommander de suivre l'évolution des autres ravageurs en cas de production industrielle de $H$. suaveolens.

\section{Conclusion}

Ce travail a permis d'identifier les pratiques culturales les mieux adaptées à la production massive de la biomasse fraîche de $H$. suaveolens.

Le labour avec semis direct et non labour (poquet) avec semis direct ont donné les meilleurs résultats qui sont tout de même similaires. Le non labour (poquet) avec semis direct est le système cultural le plus avantageux et le plus économique dans le cadre de la production foliaire massive de $H$. suaveolens. 


\section{REMERCIEMENTS}

Cette étude a été financée par le rectorat de l'Université d'Abomey-Calavi. Nous remercions Dr. G. Georgen (IITA, Bénin) pour avoir aidé pour l'identification de certaines espèces d'insectes. L'aide apportée par les ouvriers agricoles est appréciée.

\section{REFERENCES BIBLIOGRAPHIQUES}

Abagli AZ, Alavo TBC, Djouaka R, Ahomadégbé MA, Ahoton LE, Yayi E, Avlessi F. 2010. Taux de répulsion de différentes concentrations de l'huile essentielle de Hyptis suaveolens contre le moustique Anopheles gambiae (Diptera: Culicidae). Actes du $2^{\text {ème }}$ colloque des Sciences, Cultures et Technologies de l'Université d'Abomey-Calavi (UAC)-Bénin. (Sous presse).

Azevedo NR, Campos IF, Ferreira HD, Portes TA, Santos SC, Seraphin JC, Paula JR, Ferri PH. 2001. Chemical variability in the essential oil of Hyptis suaveolens. Phytochemistry, 57(5): 733736

Chukwujekwu JC, Smith P, Coombes PH, Mulholland DA, van Staden J. 2005. Antiplasmodial diterpenoid from the leaves of Hyptis suaveolens. $J$. Ethnopharmacol.,102(2): 295-297.

Coates WE, Ayerza RH. 1995. New crops for the Rio Bermejo Rivers Basin -
Phase II. Final Report submitted to COREBE, Buenos Aires, Argentina. $78 \mathrm{p}$.

Jaenson, TGT, Palsson K, Borg-Karlson, AK. 2006. Evaluation of extracts and oils of mosquito (Diptera: Culicidae) repellent plants from Sweden and Guinea-Bissau. Journal of Medical Entomology, 43(1): 113-11

Krishnamurthy YL, Shashikala J. 2006. Inhibition of aflatoxin B production of Aspergillus flavus, isolated from soybean seeds by certain natural plant products. Lett Appl Microbiol., 43(5): 469-74.

Scopel E. 1999. Le semis direct avec paillis de résidus dans l'ouest mexicain : une histoire d'eau? Agriculture et Développement, 21: 71-86.

Soltner D. 1994. Les Bases de la Production Végétale: Le sol (Tome 1). Ed. Sciences et Techniques Agricoles: Sainte-Gemmes-sur-Loire, France; 467p. ISBN : 2-907710-00-1

Ziegler HL, Jensen TH, Christensen J, Staerk D, Hagerstrand H, Sittie AA, Olsen CE, Staalso T, Ekpe P, Jaroszewski JW. 2002. Possible artefacts in the in vitro determination of antimalarial activity of natural products that incorporate into lipid bilayer: apparent antiplasmodial activity of dehydroabietinol, a constituent of Hyptis suaveolens. Planta Med., 68(6): 547-549. 